\title{
Mechanical injury of the anterior mitral leaflet late after transcatheter aortic valve implantation
}

\author{
Mizuki Miura ${ }^{1}$, Shinichi Shirai ${ }^{1}$, Kenichiro Murata ${ }^{2}$, \\ Masaomi Hayashi ${ }^{1}$, Nobuhiro Ito ${ }^{1}$, Kenji Ando ${ }^{1}$ \\ ${ }^{1}$ Department of Cardiology, Kokura Memorial Hospital, Kitakyushu, Japan \\ ${ }^{2}$ Department of Pathology, Kokura Memorial Hospital, Kitakyushu, Japan
}

An 85-year-old man with symptomatic severe aortic stenosis underwent transcatheter aortic valve implantation (TAVI) using a $26-\mathrm{mm}$ balloon expandable SAPIEN XT (Edwards Lifesciences,
Irvine, CA, USA). It was deployed via transapical approach under general anesthesia (Fig. 1A). He was referred to the documented center for dyspnea (New York Heart Association functional class IV)

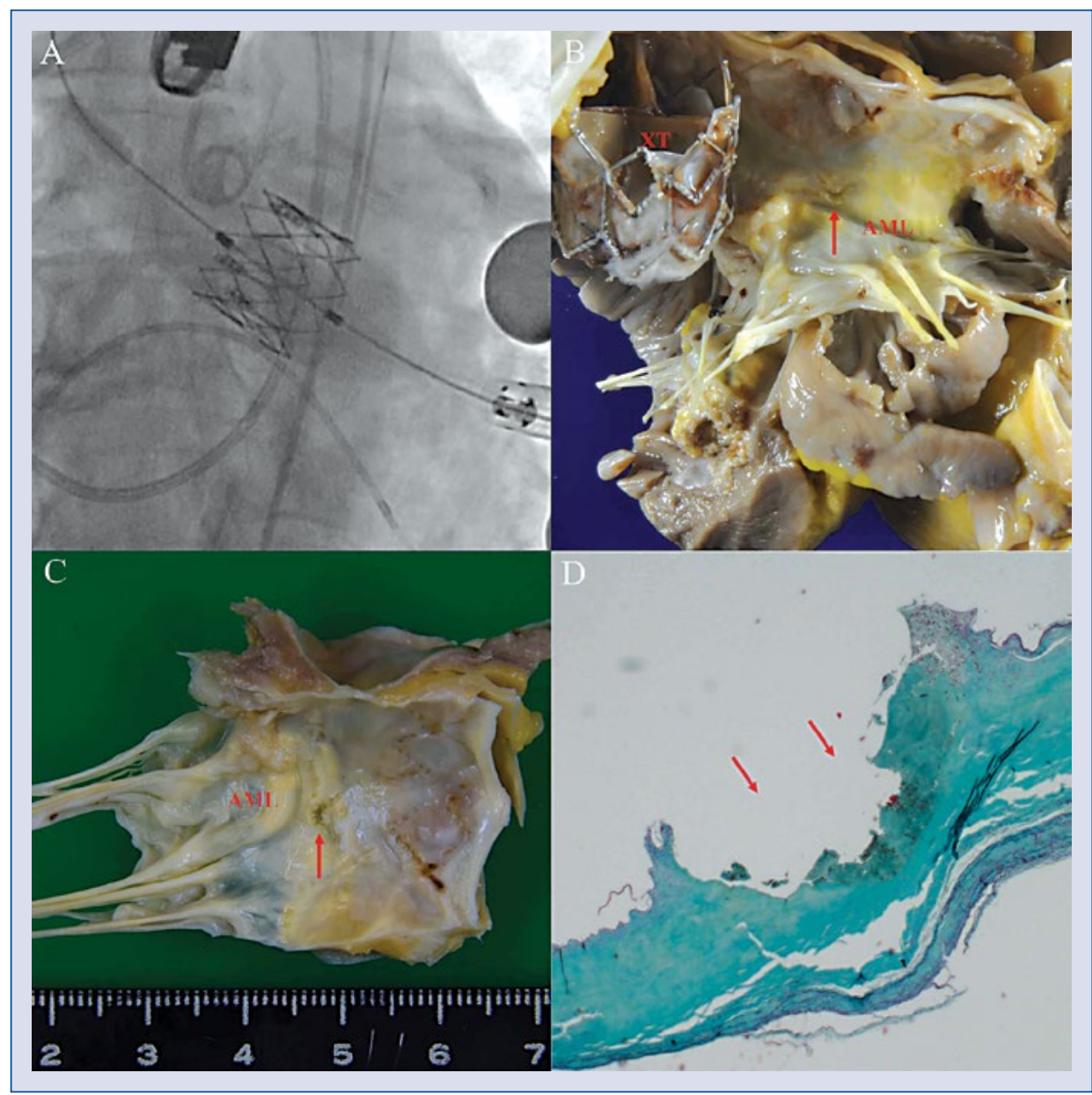

Figure 1. A. A 26-mm SAPIEN XT deployed via a transapical approach; B, C. Macroscopic findings showing the anterior mitral leaflet (AML) was injured by mechanical stimulation from the bottom of implanted valve (arrow); D. Microscopic findings showing elastic fiber injured by mechanical stimulation (arrow); XT — SAPIEN XT valve.

Address for correspondence: Mizuki Miura, MD, Department of Cardiology, Kokura Memorial Hospital, 3-2-1 Asano, Kokurakita-ku, Kitakyushu 802-8555 Japan, tel: +81-93-511-2000, fax: +81-93-511-3240, e-mail: mizumiura-circ@umin.ac.jp Received: 11.12.2017 Accepted: 29.12.2017 
14 months after TAVI. Despite intensive heart failure treatment for 1 month, he died of progressive low-output syndrome and uncontrollable heart failure. The results of autopsy clarified that he died of heart failure. Macroscopically the anterior mitral leaflet (AML) was injured with a small ulceration without vegetations (Fig. 1B, C). Microscopic findings in Elastica Masson stain surprisingly revealed focal ulceration in the ventricular side of the AML, possibly caused by mechanical stimulation rather than infectious endocarditis (Fig. 1D).

Transcatheter aortic valve implantation is an option for patients with severe symptomatic aortic stenosis at intermediate and high risk for surgical aortic valve replacement. TAVI rates have rapidly increased worldwide, but there are few reports of AML injury by mechanical stimulation. A previous study (Miura M et al., JACC Cardiovasc Intervent. 2016; 9(24): e233-e234) about perforation of AML due to mechanical stimulation late after TAVI was reported. According to available literature, this is the first report about a case of silent AML injury caused by mechanical stimulation of an implanted valve late after TAVI. It is important to avoid low implantation during the TAVI procedure. In addition, it is necessary to assess the rate of late AML injury and clinical impact of AML injury late after TAVI in the context of a large clinical study.

Conflict of interest: Dr. Mizuki Miura is a consultant for Japan Lifeline; Dr. Shinichi Shirai is proctor for transfemoral-TAVI for Edwards SAPIEN valve and Medtronic CoreValve. 